DEMOGRAPHIC RESEARCH

VOLUME 42, ARTICLE 1, PAGES 1-14

PUBLISHED 3 JANUARY 2020

https://www.demographic-research.org/Volumes/Vol42/1/

DOI: 10.4054/DemRes.2020.42.1

Descriptive Finding

Relationship between vaccination and nutritional status in children: Analysis of recent Demographic and Health Surveys

Maria Teresa Solis-Soto

Deepak Paudel

Francesco Nicoli

(C) 2020 Solis-Soto, Paudel \& Nicoli.

This open-access work is published under the terms of the Creative Commons Attribution 3.0 Germany (CC BY 3.0 DE), which permits use, reproduction, and distribution in any medium, provided the original author(s) and source are given credit.

See https://creativecommons.org/licenses/by/3.0/de/legalcode. 


\section{Contents}

1 Introduction 2

2 Data and methods 2

2.1 Data source 2

2.2 Variables definition and analysis 3

3 Results 4

4 Discussion $\quad 9$

$\begin{array}{lll}5 & \text { Acknowledgments } & 10\end{array}$

$\begin{array}{ll}\text { References } & 11\end{array}$ 


\title{
Relationship between vaccination and nutritional status in children: Analysis of recent Demographic and Health Surveys
}

\author{
Maria Teresa Solis-Soto ${ }^{1}$ \\ Deepak Paudel ${ }^{2}$ \\ Francesco Nicoli $^{3}$
}

\begin{abstract}
BACKGROUND

A body of evidence suggests that vaccines support the development of the immune system and also improve overall health.

\section{OBJECTIVE}

To study the effect of the complete basic vaccination schedule (Bacille CalmetteGuérin, i.e., BCG; measles; polio 3; and Diphtheria, Tetanus toxoids, and Pertussis, i.e., DTP3) on nutritional status of children under 2 years of age.
\end{abstract}

\section{METHODS}

Recent DHS data from 16 countries conducted after 2013 were used. After a bivariate descriptive analysis, a logistic regression analysis was conducted to predict the likelihood of underweight, stunting, and wasting by immunization status. A combined odds ratio was computed and adjusted for background variables.

\section{RESULTS}

A significantly higher prevalence of underweight was found among children with incomplete vaccination schedules in seven countries. Similarly, wasting and stunting were frequently observed in under-vaccinated children in four countries. Moreover, logistic regression adjusted for background variables revealed a relation between incomplete vaccination and underweight in Angola, Chad, and Guatemala $(95 \%$ CI lower bound $>1$ ). Combining data of all countries, underweight (adjusted Odds Ratio, aOR $1.21,95 \%$ CI $1.11-1.31$ ), wasting (aOR 1.18, 95\% CI $1.05-1.33$ ), and stunting (aOR 1.07, 95\% CI 1.00-1.14) were associated with poor vaccination status. The overall effect was consistent with both sexes except the results for wasting for females and stunting for males, though insignificant.

\footnotetext{
${ }^{1}$ Universidad de O’Higgins, Rancagua, Chile.

${ }^{2}$ Save the Children, Kathmandu, Nepal.

${ }^{3}$ Università degli Studi di Ferrara, Italy. Email: nclfnc1@unife.it.
} 


\section{CONTRIBUTION}

To our knowledge, this is the first paper assessing the relation between vaccination and nutritional status at a multi-country level with a huge dataset. Our analysis suggests a poor nutritional status in children with an incomplete vaccination schedule.

\section{Introduction}

The importance of immunizations in the decline of infant mortality has been widely recognized (Ozawa et al. 2017). Recent evidence suggests that the effect is not only due to the reduction of vaccine-preventable infections but also due to 'heterologous effects' on other diseases (Flanagan et al. 2013; Higgins et al. 2016; Nicoli and Appay 2017). Vaccines not only prevent specific infections but also prompt the development of the overall immune system (MacGillivray and Kollmann 2014). In particular, live vaccines (such as BCG and measles-containing ones) favor the development of innate and adaptive arms of the immune system (Freyne, Marchant, and Curtis 2015; Kleinnijenhuis et al. 2014; Kleinnijenhuis et al. 2012). This may improve immune responses against vaccines administered at the same moment (Ota et al. 2002) or even later (Libraty et al. 2014; Ritz et al. 2013). However, the most striking effect demonstrated so far is the lower incidence of infections (Rodrigues et al. 2006; Sorup et al. 2014; Valentiner-Branth et al. 2007) and of all-cause mortality (Higgins et al. 2016; Shann 2013) in vaccinated children.

As studies assessing the heterologous effects of vaccines have been conducted in a limited number of countries, we wanted to investigate the effect of complete vaccination on the health and nutrition of children at the multi-country level. Using data from recent Demographic and Health Surveys (DHS), we related the nutritional status of children against their vaccination status.

\section{Data and methods}

\subsection{Data source}

Data from the 20 countries where DHS were conducted from 2014 to 2016 were obtained from the DHS Program website (www.dhsprogram.com). Four of them (Afghanistan, Zimbabwe, Colombia, and Egypt) were excluded, as information on immunization and/or nutritional status was not available or not in a comparable form with other countries. DHS are periodic cross-sectional surveys conducted by the 
Ministry of Health or Office of Central Bureau of Statistics of each country that collects data focusing on fertility, child mortality, immunization, maternal health, and nutritional status (Corsi et al. 2012). Surveys included in this study had a sample size ranging from 674 to 7,537 children, with response rates of above 96\% (Table 1). Trained enumerators collected information after obtaining verbal informed consent. Ethical approvals were obtained from the review board of respective countries.

Table 1: Countries, survey year, total children aged 12-24 months, and total children with immunization and nutrition information

\begin{tabular}{|c|c|c|c|c|c|c|}
\hline Region & Country & $\begin{array}{l}\text { Survey } \\
\text { year }\end{array}$ & $\begin{array}{l}\text { Population } \\
\text { mid-2017 } \\
\text { (millions) }\end{array}$ & $\begin{array}{l}\text { Response rate } \\
\text { for household } \\
\text { surveys }(\%)\end{array}$ & $\begin{array}{l}\text { Total children } \\
\text { aged } 12-24 \\
\text { months }\end{array}$ & $\begin{array}{l}\text { Children aged } \\
\text { 12-24 months } \\
\text { with complete } \\
\text { immunization } \\
\text { and nutritiona } \\
\text { information }\end{array}$ \\
\hline \multirow{10}{*}{$\begin{array}{l}\text { Sub-Saharan } \\
\text { Africa }\end{array}$} & Angola & $2015-2016$ & 28.6 & 99.0 & 5090 & 2318 \\
\hline & Chad & 2014-2015 & 14.9 & 99.0 & 6185 & 3674 \\
\hline & Ethiopia & 2016 & 105.0 & 98.0 & 3947 & 3683 \\
\hline & Ghana & 2014 & 28.8 & 99.0 & 2204 & 1091 \\
\hline & Kenya & 2014 & 49.7 & 97.0 & 7537 & 7121 \\
\hline & Lesotho & 2014 & 2.2 & 98.0 & 1227 & 561 \\
\hline & Malawi & 2015-2016 & 18.6 & 99.0 & 6491 & 2038 \\
\hline & Rwanda & 2014-2015 & 12.3 & 99.0 & 3136 & 1503 \\
\hline & Senegal & 2016 & 15.8 & 98.6 & 2323 & 2199 \\
\hline & Tanzania & 2015-2016 & 57.5 & 98.0 & 3951 & 3730 \\
\hline \multirow{4}{*}{$\begin{array}{l}\text { South and } \\
\text { Southeast } \\
\text { Asia }\end{array}$} & Bangladesh & 2014 & 164.7 & 99.0 & 3197 & 2964 \\
\hline & Cambodia & 2014 & 15.9 & 99.0 & 2828 & 1831 \\
\hline & Myanmar & 2015-2016 & 53.4 & 98.0 & 1634 & 1470 \\
\hline & Nepal & 2016 & 29.4 & 99.0 & 1953 & 953 \\
\hline $\begin{array}{l}\text { Latin America } \\
\text { and } \\
\text { Caribbean }\end{array}$ & Guatemala & $2014-2015$ & 16.9 & 99.0 & 4855 & 4753 \\
\hline $\begin{array}{l}\text { North } \\
\text { Africa/West } \\
\text { Asia/Europe }\end{array}$ & Armenia & $2015-2016$ & 3.0 & 96.0 & 674 & 612 \\
\hline Total & - & - & - & - & 57232 & 40501 \\
\hline
\end{tabular}

1 https://www.prb.org/pdf17/2017_World_Population.pdf.

\subsection{Variables definition and analysis}

Undernutrition is measured as stunting (low height for age), wasting (low weight for height), and underweight (low weight for age). Nutritional status is an important measure of overall health, and poor nutrition results in higher mortality and lower 
quality of life (Lorem, Schirmer, and Emaus 2017). Stunting is a critical variable to define children's health, as stunting is characterized by several pathologies, and it has consequences on the future growth and potential of individuals (Prendergast and Humphrey 2014). Wasting is more reflective of acute undernutrition primarily due to short-term health issues (Saaka and Galaa 2016).

The primary dependent variables of the study are undernutrition measured as underweight, stunting, and wasting among children between 12 and 24 months of age. Any child below two standard deviations (-2SD) of reference weight for their specific age according to the World Health Organization (WHO) standard is classified as 'underweight.' Any child below $-2 \mathrm{SD}$ of reference height for their specific age according to the WHO standard is classified as 'stunted.' Any child below -2SD of reference weight for their specific height according to the WHO standard is classified as 'wasted.' Children were analyzed by sex: both sexes combined, males, and females.

The independent variable of the study is immunization status, measured with reference to basic vaccination. Basic vaccination was categorized as 'complete' if all eight basic vaccines (BCG, measles, polio 1-2-3, and DTP 1-2-3) were received by a child.

After a bivariate analysis, a logistic regression to predict the likelihood of undernutrition based on immunization status was conducted. The analysis was then adjusted for age, place of living, sex of the child, wealth quintile, mother's education, breastfeeding, and perceived weight at birth. The odds of underweight, stunting, and wasting as a result of vaccination status were compared between countries.

Bivariate and regression analysis was done using SPSS 17, and pooled analysis [meta-analysis, including test of heterogeneity (Higgins et al. 2003)] was computed using Stata SE 12. The analysis was adjusted for sampling weights and survey design for each country, as recommended by DHS.

\section{Results}

We analyzed data from 40,501 children between 12 to 24 months of age in 16 countries. As shown in Table S-1, mothers were mostly living in rural areas, literate, and breastfeeding, and around $45 \%$ were from poor families. Underweight rates were between 0.6 and $34.2 \%$, stunting rates between 7.8 and $51.7 \%$, and wasting rates between 0.9 and 15.2\%. Coverage of BCG, measles, polio ( 3 doses), and DTP ( 3 doses) vaccinations were above $90 \%$ in $12,4,3$, and 7 countries respectively. The percentage of children with a complete basic vaccination schedule was between $25.2 \%(\mathrm{Chad})$ and 86.5\% (Armenia). 
Generally, the bivariate analysis showed higher prevalence of underweight children among those with incomplete basic vaccinations, and the difference was statistically significant in Angola, Chad, Ethiopia, Guatemala, Kenya, Myanmar, and Senegal (Table 2). Similarly, stunting was significantly more prevalent in children with incomplete vaccinations in Angola, Bangladesh, Chad, and Guatemala, and wasting in Angola, Chad, Kenya, and Myanmar respectively.

Table 2: Underweight, stunting, and wasting by vaccination status

\begin{tabular}{|c|c|c|c|c|c|}
\hline & \multicolumn{5}{|c|}{ Basic vaccination schedule $^{1}$} \\
\hline & \multicolumn{5}{|c|}{ Underweight } \\
\hline & \multicolumn{2}{|c|}{ Complete } & \multicolumn{2}{|c|}{ Incomplete } & \multirow[b]{2}{*}{ p Value } \\
\hline & n Total & n Underw. (\%) & n Total & n Underw. (\%) & \\
\hline Angola & 689 & $88(12.8)$ & 1629 & $376(23.1)$ & $<0.001$ \\
\hline Armenia & 529 & $4(0.7)$ & 83 & $0(0.0)$ & 0.550 \\
\hline Bangladesh & 2537 & $854(33.7)$ & 423 & $159(37.5)$ & 0.280 \\
\hline Cambodia & 1418 & $315(22.2)$ & 412 & $108(26.2)$ & 0.228 \\
\hline Chad & 921 & $213(23.1)$ & 2730 & $1031(37.8)$ & $<0.001$ \\
\hline Ethiopia & 1386 & $298(21.5)$ & 2297 & $612(26.6)$ & 0.019 \\
\hline Ghana & 841 & $119(14.2)$ & 249 & $31(12.3)$ & 0.521 \\
\hline Guatemala & 3595 & $508(14.1)$ & 1159 & $205(17.7)$ & 0.016 \\
\hline Kenya & 5189 & $573(11.0)$ & 1911 & $261(13.7)$ & 0.015 \\
\hline Lesotho & 434 & $50(11.4)$ & 128 & $9(6.9)$ & 0.184 \\
\hline Malawi & 1530 & $152(9.9)$ & 508 & $64(12.7)$ & 0.146 \\
\hline Myanmar & 887 & $147(16.5)$ & 582 & $137(23.5)$ & 0.004 \\
\hline Nepal & 750 & $215(28.6)$ & 203 & $68(33.6)$ & 0.266 \\
\hline Rwanda & 632 & $67(10.6)$ & 871 & $91(10.4)$ & 0.908 \\
\hline Senegal & 1533 & $207(13.5)$ & 666 & $118(17.8)$ & 0.025 \\
\hline \multirow[t]{5}{*}{ Tanzania } & 2842 & $398(14.0)$ & 888 & $153(17.3)$ & 0.056 \\
\hline & \multicolumn{5}{|c|}{ Basic vaccination schedule $^{1}$} \\
\hline & \multicolumn{5}{|c|}{ Stunting } \\
\hline & \multicolumn{2}{|c|}{ Complete } & \multicolumn{2}{|c|}{ Incomplete } & \\
\hline & n Total & n Stunt. (\%) & n Total & n Stunt. (\%) & p Value \\
\hline Angola & 689 & $259(37.6)$ & 1629 & $817(50.2)$ & $<0.001$ \\
\hline Armenia & 529 & $40(7.6)$ & 83 & $8(9.2)$ & 0.650 \\
\hline Bangladesh & 2537 & $969(38.2)$ & 423 & $208(49.2)$ & 0.001 \\
\hline Cambodia & 1418 & $463(32.7)$ & 412 & $164(39.9)$ & 0.052 \\
\hline Chad & 921 & 395 (42.9) & 2730 & $1349(49.4)$ & 0.012 \\
\hline Ethiopia & 1386 & $589(42.5)$ & 2297 & 1059 (46.1) & 0.185 \\
\hline Ghana & 841 & $175(20.8)$ & 249 & 67 (26.9) & 0.109 \\
\hline Guatemala & 3595 & $1821(50.7)$ & 1159 & $635(54.8)$ & 0.045 \\
\hline Kenya & 5189 & $1645(31.7)$ & 1911 & $651(34.0)$ & 0.141 \\
\hline Lesotho & 434 & $156(35.9)$ & 128 & $53(41.5)$ & 0.276 \\
\hline Malawi & 1530 & $614(40.1)$ & 508 & $212(41.7)$ & 0.606 \\
\hline Myanmar & 887 & $267(30.1)$ & 582 & $207(35.5)$ & 0.066 \\
\hline Nepal & 750 & $312(41.6)$ & 203 & $80(39.5)$ & 0.647 \\
\hline Rwanda & 632 & $286(45.2)$ & 871 & $403(46.3)$ & 0.716 \\
\hline Senegal & 1533 & $307(20.0)$ & 666 & $160(24.0)$ & 0.063 \\
\hline Tanzania & 2842 & $1167(41.1)$ & 888 & 372 (41.9) & 0.722 \\
\hline
\end{tabular}


Table 2: (Continued)

\begin{tabular}{lccccc}
\hline & \multicolumn{5}{c}{ Basic vaccination schedule } \\
\cline { 2 - 6 } & \multicolumn{5}{c}{ Wasting } \\
\cline { 2 - 6 } & $\mathbf{n}$ Total & $\begin{array}{c}\text { Complete } \\
\text { n Wast. (\%) }\end{array}$ & n Total & Incomplete \\
n Wast. (\%) & p Value \\
\hline Angola & 689 & $22(3.2)$ & 1629 & $111(6.8)$ & 0.012 \\
Armenia & 529 & $15(2.7)$ & 83 & $3(3.6)$ & 0.701 \\
Bangladesh & 2537 & $357(14.1)$ & 423 & $56(13.3)$ & 0.710 \\
Cambodia & 1418 & $136(9.6)$ & 412 & $38(9.3)$ & 0.876 \\
Chad & 921 & $95(10.4)$ & 2730 & $461(16.9)$ & $<0.001$ \\
Ethiopia & 1386 & $144(10.4)$ & 2297 & $262(11.4)$ & 0.501 \\
Ghana & 841 & $51(6.1)$ & 249 & $9(3.5)$ & 0.135 \\
Guatemala & 3595 & $31(0.9)$ & 1159 & $12(1.0)$ & 0.684 \\
Kenya & 5189 & $190(3.7)$ & 1911 & $99(5.2)$ & 0.026 \\
Lesotho & 434 & $16(3.8)$ & 128 & $5(3.6)$ & 0.954 \\
Malawi & 1530 & $39(2.6)$ & 508 & $17(3.4)$ & 0.385 \\
Myanmar & 887 & $52(5.9)$ & 582 & $54(9.3)$ & 0.031 \\
Nepal & 750 & $65(8.7)$ & 203 & $26(13.0)$ & 0.144 \\
Rwanda & 632 & $14(2.2)$ & 871 & $21(2.4)$ & 0.851 \\
Senegal & 1533 & $106(6.9)$ & 666 & $62(9.3)$ & 0.051 \\
Tanzania & 2842 & $108(3.8)$ & 888 & $47(5.3)$ & 0.088 \\
\hline
\end{tabular}

${ }^{1}$ Include: BCG, measles, polio (3 doses), DTP (3 doses).

The effects on underweight were confirmed by the adjusted logistic regression model in Angola, Chad, and Guatemala (Figure 1), where a higher likelihood of underweight in children with the incomplete basic vaccination schedule was observed. Interestingly, these three countries have a different percentage of underweight in children $(20 \%, 34 \%$, and $15 \%$ respectively), suggesting that the variability between countries in anthropometric measures does not affect the strength of the association. However, the model did not confirm any of the associations between vaccination schedule and stunting. Interestingly, in Chad and Myanmar, higher probabilities of wasting in children with the incomplete basic vaccination schedule were observed. Notably, the meta-analysis of different countries revealed an association between incomplete vaccination and underweight (aOR 1.21, 95\% CI 1.11-1.31) as well as wasting (aOR 1.18, 95\% CI 1.05-1.33) but not for the stunting (aOR 1.07, 95\% CI $1.00-1.14)$. Heterogeneity only was statistically significant $(\mathrm{p}<0.05)$ for underweight analysis (for both sexes combined). 
Figure 1: Adjusted Odd Ratios (aORs) for underweight, stunting, and wasting in children with an incomplete basic vaccination schedule
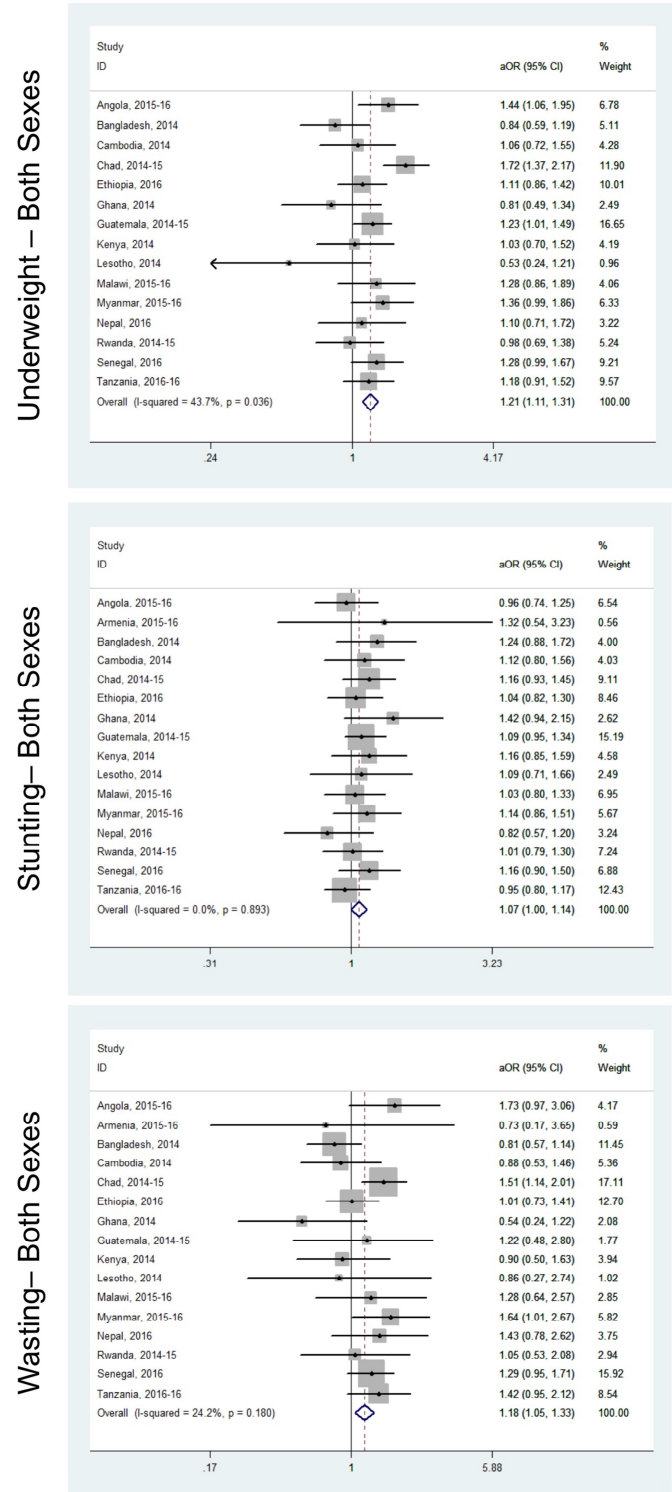
Solis-Soto, Paudel \& Nicoli: Relationship between vaccination and nutritional status in children

Figure 1: (Continued)

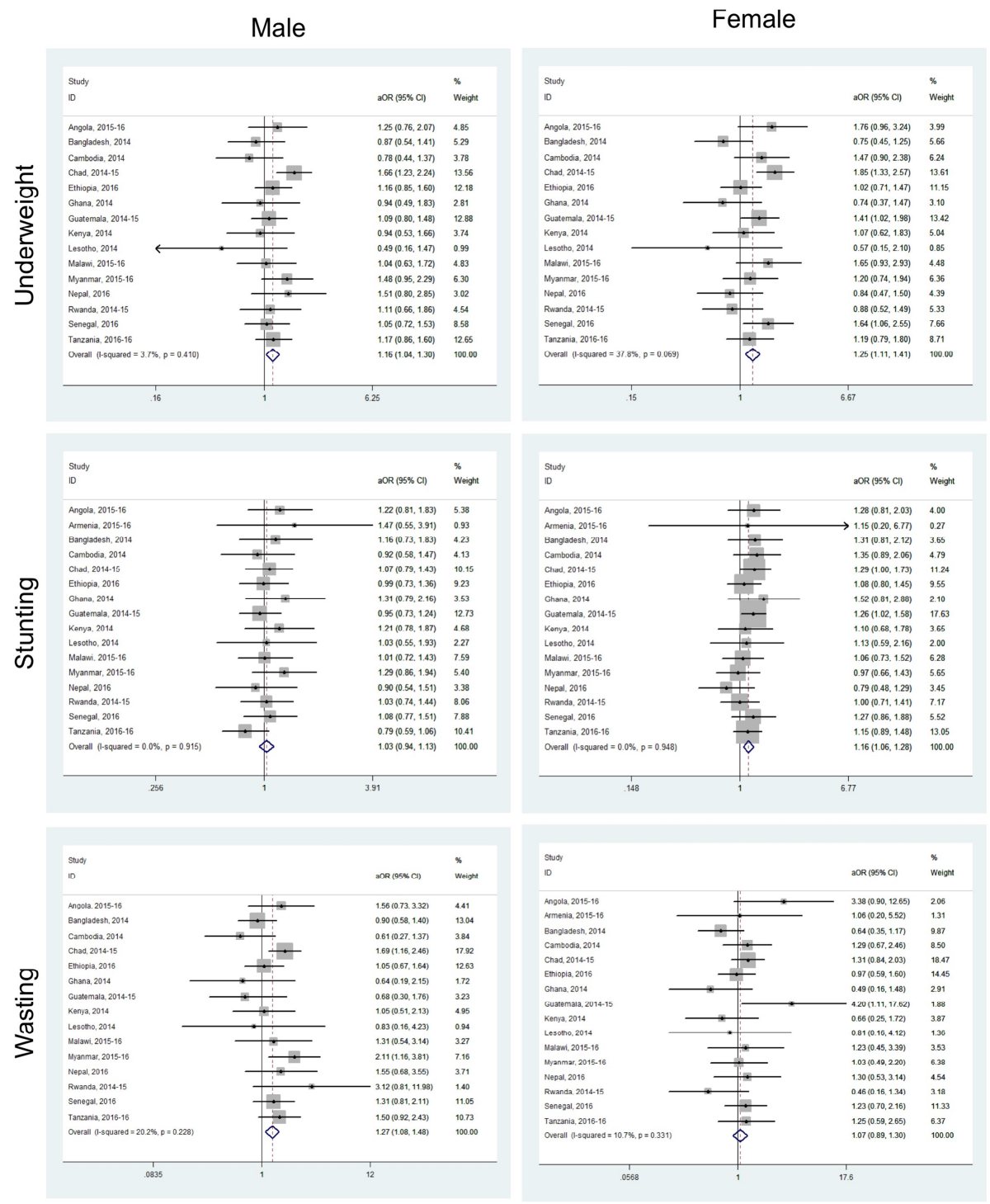

Notes: Adjusted model controls for age, place of living, sex of child, wealth quintile, mother's education, breastfeeding, and perceived weight at birth. Bars represent $95 \%$ confidence interval. Results are presented combining both sexes after stratification for sex. 
We also analyzed results for sex-specific effects of a complete immunization schedule on nutritional status. In both male and female groups, the meta-analysis revealed an overall higher probability of underweight in children with an incomplete basic vaccination schedule (male aOR 1.16, 95\% CI 1.04-1.30; female aOR 1.25, 95\% CI 1.11-1.41). However, stunting was associated with an incomplete basic vaccination schedule in females (aOR 1.16, 95\% CI 1.06-1.28) but not significant for males (aOR $1.03,95 \%$ CI $0.94-1.13$ ). Wasting was associated with an incomplete basic vaccination schedule only in males (aOR for the meta-analysis $1.27,95 \%$ CI $1.08-1.48$ ).

\section{Discussion}

In this study, we analyzed the heterologous vaccine effects on overall health as measured by underweight, stunting, and wasting in children, using DHS data from 16 countries representing different continents. As previous studies of non-specific effects of vaccines were usually performed in single countries, we deliberately chose to analyze countries with different characteristics to see if the effect could be generalized or rather associated to country-specific features. Overall, the meta-analysis showed a higher likelihood of poor nutritional status in children with incomplete immunization, as reported previously from India (Anekwe and Kumar 2012). Other reports have shown reduced all-cause mortality (Kristensen, Aaby, and Jensen 2000; McGovern and Canning 2015) as well as reduced morbidity (Otto et al. 2000) and antibiotic use (Wilby and Werry 2012) in fully immunized children, suggesting that immunization not only helps to prevent specific disease of focus but also leads to overall improvements in health.

Interestingly, after stratification for sex, all results showed that incomplete vaccinations led to higher underweight, stunting, and wasting, and the results were statistically significant except for male children for stunting, and female children for wasting. Some sex-specific differences in heterologous effects of vaccines have been postulated, usually in favor of female children (Sankoh et al. 2014). Further studies and different experimental approaches are needed to confirm and better investigate sexspecific associations for wasting and stunting.

One of the limitations of the study is related to the cross-sectional design, with the exposure and outcome being measured simultaneously, thus it is not possible to determine causality, mainly because we cannot assure temporality. In this sense, the presence of reverse causality is possible. In respect to this, we cannot exclude the 'healthy vaccine bias' or, more in general, to the 'healthy user effect.' It has indeed been demonstrated that healthy individuals usually tend to be vaccinated more frequently as well as to undertake other preventive behaviors, and these biases have also 
been suggested to affect several vaccine effectiveness studies (Remschmidt, Wichmann, and Harder 2015; Shrank, Patrick, and Brookhart 2011). Despite the fact that we adjusted for a number of variables that may, directly or indirectly, be connected with health, we cannot exclude this hypothesis and, therefore, the interpretation of our results that children with good nutritional status tend to be vaccinated more frequently should be considered while interpreting the data. We could not control the results for some variables that may be related to nutritional status and immunization, such as those related to child morbidity conditions or serum antibody levels, as such data were not collected in DHS. In addition, both the nutritional and vaccination status of children could be an indication of the 'wealth' of the health system in specific countries. However, in our study, the frequency of children with incomplete vaccination status did not significantly correlate with the percentage of undernourished children (data not shown). Thus, our analysis rather supports previous reports indicating that basic vaccinations do not harm children and their health, but complete immunization status shows a positive effect on overall health and nutrition. Since our data cover countries from different continents, we can also conclude that this is not dependent on the geographical location.

\section{Acknowledgments}

This study was supported by the 2016 Network Funds through the Center for International Health of the Ludwig Maximilians University $\left(\mathrm{CIH}^{\mathrm{LMU}}\right)$. $\mathrm{CIH}^{\mathrm{LMU}}$ is funded by the Higher Education Excellence in Development Cooperation (Exceed) program of the German Academic Exchange Service (DAAD) and the Federal Ministry for Economic Cooperation and Development (BMZ) - Germany.

Funding sources had no role in study design, analysis, and interpretation of the data, writing of the report, or in the decision to submit the article for publication. 


\section{References}

Anekwe, T.D. and Kumar, S. (2012). The effect of a vaccination program on child anthropometry: evidence from India's Universal Immunization Program. Journal of Public Health (Oxf) 34(4): 489-497. doi:10.1093/pubmed/fds032.

Corsi, D.J., Neuman, M., Finlay, J.E., and Subramanian, S.V. (2012). Demographic and health surveys: A profile. International Journal of Epidemiology 41(6): 16021613. doi:10.1093/ije/dys184.

Flanagan, K.L., van Crevel, R., Curtis, N., Shann, F., Levy, O., and Optimmunize Network (2013). Heterologous ("nonspecific") and sex-differential effects of vaccines: Epidemiology, clinical trials, and emerging immunologic mechanisms. Clinical Infectious Diseases 57(2): 283-289. doi:10.1093/cid/cit209.

Freyne, B., Marchant, A., and Curtis, N. (2015). BCG-associated heterologous immunity, a historical perspective: Experimental models and immunological mechanisms. Transactions of The Royal Society of Tropical Medicine and Hygiene 109(1): 46-51. doi:10.1093/trstmh/tru196.

Higgins, J.P., Soares-Weiser, K., Lopez-Lopez, J.A., Kakourou, A., Chaplin, K., Christensen, H., Martin, N.K., Sterne, J.A., and Reingold, A.L. (2016). Association of BCG, DTP, and measles containing vaccines with childhood mortality: Systematic review. BMJ 355: i5170. doi:10.1136/bmj.i5170.

Higgins, J.P., Thompson, S.G., Deeks, J.J., and Altman, D.G. (2003). Measuring inconsistency in meta-analyses. BMJ 327(7414): 557-560. doi:10.1136/bmj.327. 7414.557.

Kleinnijenhuis, J., Quintin, J., Preijers, F., Benn, C.S., Joosten, L.A., Jacobs, C., van Loenhout, J., Xavier, R.J., Aaby, P., van der Meer, J.W., van Crevel, R., and Netea, M.G. (2014). Long-lasting effects of BCG vaccination on both heterologous Th1/Th17 responses and innate trained immunity. Journal of Innate Immunity 6(2): 152-158. doi:10.1159/000355628.

Kleinnijenhuis, J., Quintin, J., Preijers, F., Joosten, L.A., Ifrim, D.C., Saeed, S., Jacobs, C., van Loenhout, J., de Jong, D., Stunnenberg, H.G., Xavier, R.J.., van der Meer, J.W.M., van Crevel, R., and Netea, M.G. (2012). Bacille Calmette-Guerin induces NOD2-dependent nonspecific protection from reinfection via epigenetic reprogramming of monocytes. PNAS 109(43): 17537-17542. doi:10.1073/pnas. 1202870109. 
Kristensen, I., Aaby, P., and Jensen, H. (2000). Routine vaccinations and child survival: Follow up study in Guinea-Bissau, West Africa. BMJ 321(7274): 1435-1438. doi:10.1136/bmj.321.7274.1435.

Libraty, D.H., Zhang, L., Woda, M., Acosta, L.P., Obcena, A., Brion, J.D., and Capeding, R.Z. (2014). Neonatal BCG vaccination is associated with enhanced T-helper 1 immune responses to heterologous infant vaccines. Trials in Vaccinology 3: 31-35. doi:10.1016/j.trivac.2013.11.004.

Lorem, G.F., Schirmer, H., and Emaus, N. (2017). What is the impact of underweight on self-reported health trajectories and mortality rates? A cohort study. Health and Quality of Life Outcomes 15(191). doi:10.1186/s12955-017-0766-x.

MacGillivray, D.M. and Kollmann, T.R. (2014). The role of environmental factors in modulating immune responses in early life. Frontiers in Immunology 5: 434. doi:10.3389/fimmu.2014.00434.

McGovern, M.E. and Canning, D. (2015). Vaccination and all-cause child mortality from 1985 to 2011: Global evidence from the Demographic and Health Surveys. American Journal of Epidemiology 182(9): 791-798. doi:10.1093/aje/kwv125.

Nicoli, F. and Appay, V. (2017). Immunological considerations regarding parental concerns on pediatric immunizations. Vaccine 35(23): 3012-3019. doi:10.1016/ j.vaccine.2017.04.030.

Ota, M.O., Vekemans, J., Schlegel-Haueter, S.E., Fielding, K., Sanneh, M., Kidd, M., Newport, M.J., Aaby, P., Whittle, H., Lambert, P.H., McAdam, K.P.W.J., Siegrist, C.-A., and Marchant, A. (2002). Influence of Mycobacterium bovis bacillus Calmette-Guerin on antibody and cytokine responses to human neonatal vaccination. Journal of Immunology 168(2): 919-925. doi:10.4049/jimmunol. 168.2.919.

Otto, S., Mahner, B., Kadow, I., Beck, J.F., Wiersbitzky, S.K., and Bruns, R. (2000). General non-specific morbidity is reduced after vaccination within the third month of life: The Greifswald study. Journal of Infection 41(2): 172-175. doi:10.1053/jinf.2000.0718.

Ozawa, S., Clark, S., Portnoy, A., Grewal, S., Stack, M.L., Sinha, A., Mirelman, A., Franklin, H., Friberg, I.K., Tam, Y.,Walker, N., Clark, A., Ferrari, M., Suraratdecha, C, Sweet, S., Goldie, S.J., Garske, T., Li, M., Hansen, P.M., Johnson, H.L., and Walker, D. (2017). Estimated economic impact of vaccinations in 73 low- and middle-income countries, 2001-2020. Bulletin of the World Health Organization 95(9): 629-638. doi:10.2471/BLT.16.178475. 
Prendergast, A.J. and Humphrey, J.H. (2014). The stunting syndrome in developing countries. Paediatrics and International Child Health 34(4): 250-265. doi:10.1179/2046905514Y.0000000158.

Remschmidt, C., Wichmann, O., and Harder, T. (2015). Frequency and impact of confounding by indication and healthy vaccinee bias in observational studies assessing influenza vaccine effectiveness: A systematic review. BMC Infectious Diseases 15(429). doi:10.1186/s12879-015-1154-y.

Ritz, N., Mui, M., Balloch, A., and Curtis, N. (2013). Non-specific effect of Bacille Calmette-Guerin vaccine on the immune response to routine immunisations. Vaccine 31(30): 3098-3103. doi:10.1016/j.vaccine.2013.03.059.

Rodrigues, A., Fischer, T.K., Valentiner-Branth, P., Nielsen, J., Steinsland, H., Perch, M., Garly, M.L., Molbak, K., and Aaby, P. (2006). Community cohort study of rotavirus and other enteropathogens: Are routine vaccinations associated with sex-differential incidence rates? Vaccine 24(22): 4737-4746. doi:10.1016/ j.vaccine.2006.03.033.

Saaka, M. and Galaa, S.Z. (2016). Relationships between wasting and stunting and their concurrent occurrence in Ghanaian preschool children. Journal of Nutrition and Metabolism 2016(4654920). doi:10.1155/2016/4654920.

Sankoh, O., Welaga, P., Debpuur, C., Zandoh, C., Gyaase, S., Poma, M.A., Mutua, M.K., Hanifi, S.M., Martins, C., Nebie, E., Kagoné, M., Emina, J.B., and Aaby, P. (2014). The non-specific effects of vaccines and other childhood interventions: The contribution of INDEPTH Health and Demographic Surveillance Systems. International Journal of Epidemiology 43(3): 645-653. doi:10.1093/ije/dyu101.

Shann, F. (2013). Nonspecific effects of vaccines and the reduction of mortality in children. Clinical Therapeutics 35(2): 109-114. doi:10.1016/j.clinthera.2013. 01.007 .

Shrank, W.H., Patrick, A.R., and Brookhart, M.A. (2011). Healthy user and related biases in observational studies of preventive interventions: A primer for physicians. Journal of General Internal Medicine 26(5): 546-550. doi:10.1007/ s11606-010-1609-1.

Sorup, S., Benn, C.S., Poulsen, A., Krause, T.G., Aaby, P., and Ravn, H. (2014). Live vaccine against measles, mumps, and rubella and the risk of hospital admissions for nontargeted infections. JAMA 311(8): 826-835. doi:10.1001/jama.2014.470. 
Valentiner-Branth, P., Perch, M., Nielsen, J., Steinsland, H., Garly, M.L., Fischer, T.K., Sommerfelt, H., Molbak, K., and Aaby, P. (2007). Community cohort study of Cryptosporidium parvum infections: sex-differential incidences associated with BCG and diphtheria-tetanus-pertussis vaccinations. Vaccine 25(14): 2733-2741. doi:10.1016/j.vaccine.2006.01.035.

Wilby, K.J. and Werry, D. (2012). A review of the effect of immunization programs on antimicrobial utilization. Vaccine 30(46): 6509-6514. doi:10.1016/j.vaccine. 2012.08.031. 